\title{
A Participação da Mulher no Setor Saúde no Brasil - 1970/80
}

\author{
* Maria Helena Machado
}

* Socióloga, Pesquisadora do DMPS-UFMG

\section{INTRODUÇÃO}

No período entre 1940 e 1970, o crescimento demográfico no Brasil registrou índices exemplares. Entre 1950 e 1960 a força de trabalho urbana cresceu em 9,2 milhões de trabalhadores. Porém, sabe-se que este crescimento não acompanhou os ganhos qualitativos na vida dessa população. Costa ${ }^{2}$ salienta que em 1970 somente $10,6 \%$ da força de trabalho urbana encontravam-se no setor primário; $25 \%$ contaram com apenas seis anos de escolaridade; 38,2\% encontravam-se na faixa etária de 15 a 19 anos e a participação feminina neste período, atingia apenas $22,5 \%$ da PEA.

Outro dado importante é que, em 1983, 43,39\% dos trabalhadores, ocupados no Brasil, ganhavam até 1 salário-mínimo e a soma de todos os trabalhadores que recebiam até 5 salários-mínimos atingia o percentual de 87,9\%.

Em 1980 registrou-se uma elevada participação da mãode-obra brasileira no setor terciário, ou seja, enquanto o setor primário absorveu 29,8\% o terciário absorveu 45,6\%.

Em estudos recentes, Nogueira e Médici ${ }^{5}$ apontam que, no periodo de $1950 / 1980$, houve aumento de 26,4 para $46,9 \%$ do total de pessoas ocupadas que atuam no setor terciário, revelando uma tendência à terciarização da economia brasileira.

O setor saúde representou neste período o segmento da economia que mais cresceu. Entre 1976 e 1982 o emprego de saúde teve um incremento de $145 \%$.

O Brasil em 1976 contava com 13.133 estabelecimento de saúde, sendo que destes, 6.368 eram privados $(48,5 \%)$ e 6.765 eram públicos $(51,5 \%)$. Estes estabelecimentos ofereciam um total de 349.386 empregos.

Já em 1980, o Brasil contava com 18.489 estabelecimentos de saúde, sendo que destes, 10.045 eram públicos $(54,32 \%)$ e $8.444(45,68 \%)$ eram privados.

Dos 346.295 empregos existentes em 1976, 138.687 eram de profissionais de nivel superior; 141.011 de nfvel elementar e 66.597 de nível médio; o que representa 40,05, 40,72 e $19,23 \%$ dos empregos de saúde, respectivamente.

Os empregos de nivel superior em 1982, passam para 228.228 (41,11\%); os de nível médio para $122.376(22,05 \%)$ e os de nível elementar para 204.537 (36,84\%).

Dos empregos de profissionais de nivel superior, $76,05 \%$ são representados por médicos. Os empregos de auxiliares de 
* Dados retirados do relatório parcial da pesquisa: Dinâmica do emprego em saúde no Brasil 1976/1983. OPS/IBGE/ENSP/

FMG, 1986.

* * - Para maiores crificas ver: BOAVENTURA, J. \& MACHADO, M.H. As formas do trabalho médico em Belo Horizonte - 1940/1979. Relatório de pesquisa Belo Horizonte, 1983. mimeo.

MÉDICI, A.C. Estrutura e dinámica da força de trabatho médico no Brasil na dé. cada de 70, RAP, Rio de Janeiro, 1985.

* Em especial para as atividades ligadas d saúde, educação $e$ prestação de serviços.

* * Para maiores critica ver: BOAVENTURA, J. \& $M A C H A D O, M . A$. A mulher médica e o mercado de trabalho. Cadernos do Internato Rural, 2 $(3 / 4) ;, 1983$. enfermagem representam nada menos que $66 \%$ do contingente de empregos de nível médio. $\mathrm{E}$ os empregos de atendentes representam cerca de $90 \%$ dos empregos de nível elementar.*

Segundo Nogueira, 4 , "entre os profissionais de saúde, a expansão foi impulsionada mais pelos profissionais de nível superior (taxa de 130,04\%) do que pelos níveis médio e elementar (taxa de 89,07\%). Trata-se de um dado associado ao "boom" do ensino superior verificado entre 1965 e 1975."

Neste sentido, os médicos representam a modalidade profissional de nível superior que mais cresceu nestes últimos anos. Houve um crescimento bruto da ordem de $126 \%$, passando de 45.000 para 102.000 médicos, neste período*. (Estima-se para 1985 a existência de 130.000 profissionais médicos no Brasil).

Resumindo, pode-se dizer que: no período de 1970-80 houve aumento significativo dos enfermeiros $(142,9 \%)$ e a categoria médica cresceu tanto quanto o pessoal de enfermagem de nivel médio e elementar, totalizando $85 \%$ dos profissionais de saúde que atuam diretamente no setor.

\section{A MULHER NO MERCADO DE TRABALHO}

A participação de força de trabalho feminina na esfera produtiva, nos últimos anos, é cada vez mais expressiva. "A inserção da mulher no mercado de trabalho, após a abolição da escravatura, alterou-se ao longo das décadas. Em 1940 o contigente feminino era de $19 \%$ do total; em 1950, 14\%; em 1960, 13\%; em 1970 cresceu para $20 \%$ e em 1980 o contingente feminino passou a representar $38,4 \%^{3}$. Contudo, ressalta-se que, a participação da mão-de-obra feminina se deu, majoritariamente, no setor terciário da economia*. Sabe-se também que as atividades vinculadas a esse setor são, quase sempre de baixo prestígio e de menores rendimentos. Por exemplo, os serviços domésticos remunerados representam uma parcela significativa das trabalhadoras brasileiras. Em 1970 havia cerca de 1.700 .000 mulheres que se ocupavam dessas atividades.

Outro dado bastante relevante é a situação salarial dessas trabalhadoras. Em 1980, de um total de 7.564.681 mulheres economicamente ativas, $51 \%$ ganhavam até 1 salário mínimo; enquanto que, apenas $7,7 \%$ ganhavam entre 10 a 20 salários mínimos.

São várias as restrições, discriminações e reduzidas as chances que as mulheres têm quando de sua inserção no mercado de trabalho.

Em via de regra, acabam por procurar profissiões, ramos de atividades, ou ocupações que não provoquem um rompimento radical com suas atividades no âmbito doméstico. E o caso, por exemplo, das mulheres médicas, que acabam, de uma forma ou de outra, direcionando sua carreira profissional para as especialidades de pediatria, ginecologia, psicoterapia, etc.* 
Pode-se dizer que essas mulheres fazem de seu trabalho uma extensão do doméstico. As tarefas de lidar com crianças (pediatria, professora maternal/primário), o cuidar de enfermos (enfermeiros, técnicos de enfermagem), os serviços domésticos e prestações de serviços (atendentes de enfermagem, domésticas, serventes, faxineiras, costureiras, etc.), são, em via de regra, executadas por mulheres. Em alguns momentos, justifica-se tais tarefas executadas por mulheres, como sendo "tarefas femininas", onde se "supōe" que elas as exerceriam com maior "eficiência" que os homens.

Os setores de saúde, educação, lazer, enfim, setores sociais, são exemplos típicos desta situação. A força de trabalho que atua nestes setores é constituída, majoritariamente, de mulheres.

Ao deter-se na análise dos dados do Censo relativos a força de trabalho em saúde (FTS) verifica-se um incremento expressivo da força de trabalho feminina, ou seja, um franco processo de feminilização do setor. (Ver tabela 1)

TABELA 1

DISTRIBUIÇÃO DOS PROFISSIONAIS DE SAÚDE - BRASIL - 1970 - 8Q11)

\begin{tabular}{lccccc}
\hline \multirow{2}{*}{ ANO } & TOTAL & \multicolumn{2}{c}{ HOMEM } & \multicolumn{2}{c}{ MULHER } \\
\cline { 3 - 6 } & & ABSOLUTO & $\%$ & ABSOLUTO & $\%$ \\
\hline 1970 & 316.730 & 170.106 & 54 & 146.624 & 46 \\
1980 & 594.406 & 255.664 & 43 & 338.742 & 57 \\
\hline
\end{tabular}

Fonte: IBGE - 1980-80.

(1) Exclui outros de ntvel superior, médio e elementar

Comparando os anos de 1970 e 1980, pode-se observar ainda que houve um incremento significativo da FT feminina, empregada no setor saúde. A participação feminina passa de 46 para $57 \%$ do total de pessoas que atuavam diretamente no setor saúde.

O crescimento maior da FT feminina neste setor, se deu com os profissionais de nivel superior, que passaram de $18 \%$ em 1970 para 35\% em 1980; já com os profissionais de nivel médio e elementar, este crescimento foi um pouco inferior (de 59 para 68\%).

A ampliação das vagas nas universidades brasileiras, contribuiu decisivamente, para tal inrremento. No período de 1961/1971, o número de alunos universitários passou de 100 mil para mais de 500 mil.

A participação da mulher universitária passou a representar, em 1971, mais de $40 \%$ da população estudantil. Na área da saúde, este fenômeno pode ser observado com maior clareza, na evolução da participação dos profissionais femininas 
* No periodo de 1970/1980, as categorias médica e odontologica acusaram um crescimento de 302 e $344 \%$, respectivamente. (médicas, odontólogas) no perfodo pós-70*. Essas duas categorias, ate bem pouco tempo, eram consideradas e exercidas, quase exclusivamente, por homens. Anteriormente, raras foram as mulheres que se atreviam a exercer tais profissões. $\mathrm{Na}$ tabela 2 verifica-se a evolução da participação das mulheres neste setor. Hoje, a participação das mulheres no setor saúde, representa cerca de $70 \%$ do contingente da FTS.

\section{TABELA 2* \\ EVOLUÇÃO DA PARTICIPAÇÃO DAS MULHERES \\ SEGUNDO ALGUMAS CATEGORIAS PROFISSIONAIS DE SAÚDE - BRASIL, 1970-80}

\begin{tabular}{lrrrrrr}
\hline \multirow{2}{*}{ CATEGORIAS } & \multicolumn{2}{c}{1970} & & 1980 & \multicolumn{2}{c}{ CRESCIMENTO BRUTO } \\
\cline { 2 - 7 } & NÚMERO & $\%$ & NÚMERO & $\%$ & HOMENS & MULHERES \\
\hline PROFISSIONAIS NÍVEL & & & & & & \\
SUPERIOR & & & & & & \\
$\quad$ Médicos & 5.221 & 11,6 & 20.992 & 20,6 & 302 & 47 \\
Odontólogos & 3.495 & 11,5 & 15.542 & 28,2 & 344 & 261 \\
Farmacêuticos & 1.747 & 40,0 & 2.210 & 37,7 & 26 & \\
Enfermeiros & 4.774 & 86,0 & 11.361 & 94,0 & 138 & \\
PROFISSIONAIS NÍvEL & & & & & & \\
MÉDIO E ELEMENTAR & & & & & & \\
$\quad$ Pessoal Enfermagem & 109.836 & 81,9 & 252.253 & 84,0 & 129 & \\
Pessoal n/Enfermagem & 21.550 & 24,5 & 36.384 & 30,5 & 25 & 69 \\
\hline
\end{tabular}

Fonte: IBGE, 1970-80.

* Tabela retirada: MACHADO, M.H. A participação da mulher na força de trabalho em saúde no Brasil 1970-80, Belo Horizonte, 1986. mimeo.

* Refere-se a técnico de enfermagem, auxiliar de enfermagem (ntvel médio) e atendente (ntvel elementar).
A tendência à feminilização no setor saúde, registrada no período pós-70, configura uma situação peculiar.

Outras tendências, tais como, o rejuvenescimento do conjunto da FTS, o amento da jornada de trabalho, o assalariamento, etc., serão também aqui analisados.

Três são as categorias que constituem a base técnica deste setor: os médicos, os enfermeiros e o pessoal de enfermagem* de nível médio e elementar. Estas totalizam mais de $85 \%$ nota do conjunto da FTS.

E são exatamente essas três categorias que concentram a maior parcela da FT feminina, ou seja, 84\%, como mostra a tabela 3. Daí, o interesse em deter a análise nessas três modalidades profissionais.

A evolução da participação das mulheres médicas neste período, foi exemplar, passando de $12 \%$ em 1970 , pra $21 \%$ em 1980 , com um incremento de $302 \%$. 
TABELA 3

PROFISSIONAIS MÉDICOS, ENFERMEIROS E

PESSOAL POR ANO E SEXO - BRASIL - 1970-80

\begin{tabular}{lrrrrrr}
\hline \multirow{2}{*}{ CATEGORIA } & \multicolumn{5}{c}{1970} & \multicolumn{3}{c}{1980} \\
\cline { 2 - 7 } & HOMEM & MULHER & TOTAL & HOMEM & MULHER & TOTAL \\
\hline Médico & 39.892 & 5.221 & 45.113 & 80.801 & 20.992 & 101.793 \\
Enfermeiro & 200 & 4.774 & 4.974 & 723 & 11.361 & 12.084 \\
Pess. Enf. & 24.263 & 109.836 & 134.099 & 48.135 & 252.253 & 300.388 \\
\hline
\end{tabular}

Fonte: IBGE - 1970-80

As enfermeiras apresentaram crescimento de $138 \%$ neste período, passando de 86\% em 1970, para 94\% em 1980.

O Pessoal de Enfermagem foi o que menos cresceu, passando de 82 para $84 \%$, com incremento de $130 \%$.

Como mostra a tabela 4, a participação das mulheres médicas, enfermeiras e do pessoal de enfermagem $\varepsilon$ bastante elevada, principalmente as duas últimas categorias que são constituídas fundamentalmente de mão-de-obra feminina. A categoria médica também experimentou um crescimento exemplar da participação das mulheres nos últimos anos.

A tendência ao rejuvenescimento da FTS, configura-se uma situação recente neste setor e representa, sem dúvida, fator decisivo na composição da FTS.

TABELA 4

EVOLUÇÃO DA PARTICIPAÇÃO DAS MULHERES

MÉDICAS, ENFERMEIRAS E PESSOAL

DE ENFERMAGEM - BRASIL - 1970-80

\begin{tabular}{lccc}
\hline \multicolumn{1}{c}{ CATEGORIAS } & $\begin{array}{c}1970 \\
(\%)\end{array}$ & $\begin{array}{c}1980 \\
(\%)\end{array}$ & $\begin{array}{c}1970 / 1980 \\
(\%)\end{array}$ \\
\hline Médica & 12 & 21 & 302 \\
Enfermeira & 86 & 94 & 138 \\
Pess. Enf. & 82 & 84 & 130 \\
\hline
\end{tabular}

Fonte: IBGE-1970-80

Como pode-se notar a participação da mulheres neste momento foi fundamental para o rejuvenescimento das categorias. Essa participação se deu com maior ênfase nas categorias de nível superior (médicos e odontólogos). Outro dado interessante, $\varepsilon$ o incremento da participação feminina, na faixa etária de mais de 60 anos. Isto se explica pela presença significativa de farmacêuticas, ou seja, pelo processo de envelhecimento que essa categoria passou a experimentar a partir de 70. (Ver Tabela 5). 
TABELA 5

PROFISSIONAIS DE SAÚDE POR ANO E SEXO, SEGUNDO FAIXA ETARIA

- BRASIL - 1970-80

\begin{tabular}{lcccccc}
\hline \multicolumn{1}{r}{$\begin{array}{c}\text { TOTAL } \\
\text { GERAL }\end{array}$} & \multicolumn{2}{c}{1970} & \multicolumn{2}{c}{1980} & \multicolumn{2}{c}{ TAXA } \\
FAIXA & \multicolumn{2}{c}{ CRESCIMENTO 70/80 } \\
\cline { 2 - 7 } ETÁRIA & HOMEM & MULHER & HOMEM & MULHER & HOMEM & MULHER \\
Até 29 anos & 46.099 & 61.493 & 80.129 & 162.638 & 74 & 164 \\
30 a 39 anos & 49.436 & 43.270 & 81.145 & 106.371 & 64 & 146 \\
40 a 59 anos & 53.374 & 38.469 & 78.737 & 83.818 & 47 & 118 \\
Mais de 60 anos & 10.699 & 2.839 & 16.980 & 4.868 & 59 & 71 \\
TOTAL & 159.608 & 146.071 & 256.991 & 357.695 & 61 & 145 \\
\hline
\end{tabular}

Fonte: IBGE - 1970-80

Ao se analisar o comportamento destas três categorias típicas, verifica-se, por exemplo que: em 1970 as mulheres médicas com menos de 39 anos somavam 44\%; as enfermeiras $51 \%$ e o pessoal de enfermagem de nível médio e elementar somava $65 \%$.

Já em 1980 , estas categorias passam para 62,84 e $71 \%$ respectivamente. Em outras palavras, uma grande maioria da FT feminina dessas modalidades profissionais era constituida essencialmente de mão-de-obra jovem, como pode-se observar na Tabela 6.

TABELA 6

EVOLUÇÃO DA PARTICIPAÇÃO DS MULHERES MÉDICAS, ENFERMEIRAS E PESSOAL DE ENFERMAGEM POR ANO, SEGUNDO FAIXA ETÁRIA $-B R A S I L-1970-80$

\begin{tabular}{|c|c|c|c|c|c|c|}
\hline \multirow{2}{*}{$\begin{array}{l}\text { FAIXA } \\
\text { ETÁRIA }\end{array}$} & \multicolumn{3}{|c|}{1970} & \multicolumn{3}{|c|}{1980} \\
\hline & MÉDICA & ENFERM. & PESS. ENF. & MÉDICA & ENFERM. & PESS. ENF. \\
\hline $\begin{array}{l}\text { Até } 29 \text { anos } \\
30 \text { a } 39 \text { anos } \\
40 \text { a } 59 \text { anos } \\
+60 \text { anos } \\
\text { TOTAL }\end{array}$ & $\begin{array}{r}12 \\
32 \\
44 \\
12 \\
100\end{array}$ & $\begin{array}{l}51^{, 00} \\
49^{, 00} \\
100\end{array}$ & $\begin{array}{r}39 \\
26 \\
31 \\
04 \\
100\end{array}$ & $\begin{array}{r}18 \\
44 \\
31 \\
08 \\
100\end{array}$ & $\begin{array}{c}42 \\
42 \\
16 \\
100^{, 00}\end{array}$ & $\begin{array}{r}45 \\
26 \\
25 \\
04 \\
100\end{array}$ \\
\hline
\end{tabular}

Fonte: IBGE - 1970-80

Outra tendência da FTS, é o progressivo processo de assalariamento, pelo qual passam os profissionais de saúde de nível superior.

Esse processo evidenciou-se ainda mais nos anos 80.

Se se comparar o assaluriamento da FT feminina com a masculina, vê-se que, no primeiro caso, o assalariamento foi maior. Em 1980, enquanto $76 \%$ das mulheres eram assalaria- 
dos, os homens somavam 58\%. Todavia, verificou-se que, mais de $50 \%$ dos trabalhadores de saúde de nível superior já encontravam-se assalariados. Houve um incremento na participação das mulheres que atuam de forma autônoma, nos anos 80. Acredita-se que este incremento deveu-se, fundamentalmente, a inserção dos psicólogos, pós-70, no mercado (Estes, constituídos majoritariamente, por mulheres ).

Como pode-se observar na Tabela 7, em 1980 mais de $70 \%$ da FT médica (de ambos sexos) já se encontravam assalariados.

TABELA 7

PROFISSIONAIS MÉDICOS, ENFERMEIROS, POR ANO E SEXO, SEGUNDO POSIÇÃO NA OCUPAÇÃO - BRASIL - 1970-80

\begin{tabular}{|c|c|c|c|c|c|}
\hline \multirow{3}{*}{ OCUPAÇÃO } & \multirow{3}{*}{ POSIÇÃO } & \multicolumn{4}{|c|}{1970} \\
\hline & & \multicolumn{2}{|c|}{ MÉDICOS } & \multicolumn{2}{|c|}{ ENFERMEIROS } \\
\hline & & HOMEM & MULHER & HOMEM & MULHER \\
\hline $\begin{array}{l}\text { EMPREGADOS } \\
\text { AUTÔNOMOS } \\
\text { TOTAL }\end{array}$ & & $\begin{array}{r}66 \\
34 \\
100\end{array}$ & $\begin{array}{r}78 \\
22 \\
100\end{array}$ & $100^{, 00}$ & $100^{, 00}$ \\
\hline \multirow{3}{*}{\multicolumn{2}{|c|}{ OCUPAÇÃO }} & \multicolumn{4}{|c|}{1980} \\
\hline & & \multicolumn{2}{|c|}{ MÉDICOS } & \multicolumn{2}{|c|}{ ENFERMEIROS } \\
\hline & & HOMEM & MULHER & HOMEM & MULHER \\
\hline $\begin{array}{l}\text { EMPREGADOS } \\
\text { AUTONONOMOS } \\
\text { TOTAL }\end{array}$ & & $\begin{array}{r}72 \\
28 \\
100\end{array}$ & $\begin{array}{r}79 \\
21 \\
100\end{array}$ & $100^{, 00}$ & $\begin{array}{r}99 \\
1 \\
100\end{array}$ \\
\hline
\end{tabular}

Fonte: IBGE -1970-80

Situação semelhante ocorreu com os enfermeiros, já que a maioria encontrava-se, neste período, assalariada.

Em pesquisa realizada com as médicas minerais (1983)* verificou-se que, em grande parte, essas profissionais optaram por especialidades de pediatria, ginecologia e psiquiatria, exercendo de forma autônoma, em seus consultórios. Em geral, as justificativas para tal situação giravam em torno de: terem horário livre e flexível, não depender de patrão, condições de exercer a profissão segundo suas possibilidades pessoais e por fim, a melhor maneira de conciliar as tarefas domésticas (Mãe, esposa. etc.) com sua vida profissional.

Quanto à jornada de trabalho do conjunto da FTS, pode-se afirmar que houve um aumento de horas de trabalho semanais,

* Ver BOAVENTURA, J.e MACHADO, M. H. - Obra já citada. 
tanto do pessoal de nível superior como de nível médio e elementar, com maior ênfase para os profissionais universitários.

Conforme demonstro em trabalhos recentes, há uma disparidade entre sexos, no que se refere à jornada de trabalho. Em outras palavas, em 1980 enquanto 54,05\% da FT feminina tinham jornada de trabalho de até 49 horas; $74,77 \%$ da FT masculina tinham sua jornada de 40 a mais de 50 horas semanais. Mais especificamente, enquanto no período analisado $42,27 \%$ dos profissionais masculinos atingiram jornadas de trabalho de mais de 50 horas semanais os profissionais femininos, nesta mesma condição, somaram apenas $16,91 \%$.

Observando os dados disponíveis verifica-se um incremento maior da jornada de trabalho das médicas (que cresceu no período de $1970 / 80$, na ordem de $424 \%$, para as jornadas com mais de 50 horas semanais trabalhadas). Em 1980, 50\% dessas profissionais tinham sua jornada de trabalho acima de 50 horas semanais, conforme mostra os dados da Tabela 8. Ressalta-se aqui, que apenas $30 \%$ dos médicos encontravam-se em tal situação.

TABELA 8

EVOLUÇĀO DOS PROFISSIONAIS MÉDICOS, ENFERMEIROS

E PESSOAL DE ENFERMAGEM, POR ANO E SEXO, SEGUNDO HORAS SEMANAIS TRABALHADAS

$-B R A S I L-1970-80$

\begin{tabular}{|c|c|c|c|c|c|c|}
\hline \multirow{3}{*}{ TRABALHADAS } & \multicolumn{6}{|c|}{1970} \\
\hline & \multicolumn{2}{|c|}{ MÉDICOS } & \multicolumn{2}{|c|}{ ENFERMEIROS } & \multicolumn{2}{|c|}{ PESS. ENF. } \\
\hline & HOMEM & MULHER & HOMEM & MULHER & HOMEM & MULHER \\
\hline $\begin{array}{l}\text { Ate } 39 \text { anos } \\
40 \text { a } 49 \text { anos } \\
+50 \text { horas } \\
\text { TOTAL }\end{array}$ & $\begin{array}{r}27 \\
54 \\
22 \\
100\end{array}$ & $\begin{array}{r}16 \\
45 \\
39 \\
100\end{array}$ & $\begin{array}{r}28 \\
45 \\
27 \\
100\end{array}$ & $\begin{array}{c}49 \\
51 \\
100^{, 00}\end{array}$ & $\begin{array}{r}17 \\
62 \\
21 \\
100\end{array}$ & $\begin{array}{r}20 \\
57 \\
23 \\
100\end{array}$ \\
\hline \multirow{3}{*}{ TRABALHADAS } & \multicolumn{6}{|c|}{1980} \\
\hline & \multicolumn{2}{|c|}{ MÉDICOS } & \multicolumn{2}{|c|}{ ENFERMEIROS } & \multicolumn{2}{|c|}{ PESS. ENF. } \\
\hline & HOMEM & MULHER & HOMEM & MULHER & HOMEM & MULHER \\
\hline $\begin{array}{l}\text { Até } 39 \text { anos } \\
40 \text { a } 49 \text { anos } \\
+50 \text { horas } \\
\text { TOTAL }\end{array}$ & $\begin{array}{r}38 \\
32 \\
30 \\
100\end{array}$ & $\begin{array}{r}23 \\
27 \\
50 \\
100\end{array}$ & $\begin{array}{r}34 \\
47 \\
19 \\
100\end{array}$ & $\begin{array}{r}33 \\
61 \\
06 \\
100\end{array}$ & $\begin{array}{r}20 \\
61 \\
19 \\
100\end{array}$ & $\begin{array}{r}16 \\
59 \\
25 \\
100\end{array}$ \\
\hline
\end{tabular}

Fonte: IBGE - 1970-80

O mesmo não ocorre com os enfermeiros, já que em ambos os sexos, a maioria desses trabalha em torno de 40 horas 
semanais. Jornadas mais longas são praticadas por uma parcela reduzida da categoria (6\% do sexo feminino e $19 \%$ do sexo masculino).

Também com o pessoal de enfermagem, mais de $80 \%$ dos homens e $75 \%$ ds mulheres têm sua jornada de trabalho de até 49 horas semanais. Contudo, registram-se $25 \%$ de mulheres que trabalham mais de 50 horas por semana.

Todavia, como será visto a seguir, o fato dessas trabalhadoras terem jornadas de trabalho tão longas, não se reverte em ganhos salariais, se comparado com os salários dos profissionais masculinos. Pelo contrário, é exatamente nesta situa-

TABELA 9

EVOLUÇÃO SALARIAL DOS PROFISSIONAIS MÉDICOS, ENFERMEIROS E PESSOAL DE ENFERMAGEM, POR ANO E SEXO, - BRASIL - 1970-80

\begin{tabular}{|c|c|c|c|c|c|c|}
\hline \multirow{3}{*}{$\begin{array}{l}\text { FAIXA } \\
\text { SALARIAL }(\mathrm{sm})\end{array}$} & \multicolumn{6}{|c|}{1970} \\
\hline & \multicolumn{2}{|c|}{ MÉDICOS } & \multicolumn{2}{|c|}{ ENFERMEIROS } & \multicolumn{2}{|c|}{ PESS. ENF. } \\
\hline & HOMEM & MULHER & HOMEM & MULHER & HOMEM & MULHER \\
\hline $\begin{array}{l}\text { A té } 3 \mathrm{sm} \\
3 \text { a } 10 \mathrm{sm} \\
10 \text { a } 20 \mathrm{sm} \\
20 \text { a } 30 \mathrm{sm} \\
\text { + DE } 30 \mathrm{sm} \\
\text { TOTAL }\end{array}$ & $\begin{array}{r}2 \\
26 \\
47 \\
16 \\
9 \\
100\end{array}$ & $\begin{array}{c}10 \\
61 \\
27 \\
2 \\
100^{, 00}\end{array}$ & $\begin{array}{l}49^{, 00} \\
51 \\
100^{, 00}\end{array}$ & $\begin{array}{c}17 \\
83 \\
, 00 \\
, 00 \\
100 \\
, 00\end{array}$ & $\begin{array}{c}84 \\
15 \\
, 4 \\
, 00 \\
100\end{array}$ & $\begin{array}{c}93 \\
7 \\
, 00 \\
\text { N/A } \\
\text { N/A } \\
100\end{array}$ \\
\hline \multirow{3}{*}{$\begin{array}{l}\text { FAIXA } \\
\text { SALARIAL (sm) }\end{array}$} & \multicolumn{6}{|c|}{1980} \\
\hline & \multicolumn{2}{|c|}{ MÉDICOS } & \multicolumn{2}{|c|}{ ENFERMEIROS } & \multicolumn{2}{|c|}{ PESS. ENF. } \\
\hline & HOMEM & MULHER & HOMEM & MULHER & HOMEM & MULHER \\
\hline $\begin{array}{l}\text { Até } 3 \mathrm{sm} \\
3 \text { a } 10 \mathrm{sm} \\
10 \text { a } 20 \mathrm{sm} \\
20 \text { a } 30 \mathrm{sm} \\
+ \text { DE } 30 \mathrm{sm} \\
\text { TOTAL }\end{array}$ & $\begin{array}{r}2 \\
19 \\
35 \\
25 \\
18 \\
100\end{array}$ & $\begin{array}{r}4 \\
62 \\
26 \\
4 \\
3 \\
100\end{array}$ & $\begin{array}{r}100^{, 00} \\
, 00 \\
, 00 \\
100^{, 00}\end{array}$ & $\begin{array}{r}15 \\
78 \\
7 \\
, 00 \\
100\end{array}$ & $\begin{array}{c}77 \\
22 \\
1 \\
, 00 \\
, 00 \\
100\end{array}$ & $\begin{array}{c}85 \\
15 \\
, 00 \\
, 00 \\
100 \\
, 00\end{array}$ \\
\hline
\end{tabular}

Fonte: IBGE-1970-80

ção que configura-se a maior desigualdade entre os sexos. Os desníveis salariais entre sexos existem e são bastante significativos.

Em 1980, cerca de $70 \%$ da FT feminina em saúde recebiam salários inferiores a 3 salários mínimos. É bom salientar também que, esta disparidade dá-se com maior ênfase entre os 
* Esta questão não se esgota aqui, apenas inicia-se. É preciso analisar atraves de dados mais qualitativos, as razóes e causas desta e de outras situações de desigualdades em que se encontra uma grande parcela feminina. profissionais universitários, em especial com os odontólogos e os médicos.

Como mostram os dados da Tabela 9, enquanto em 1980, $68 \%$ das mulheres médicas recebiam salários inferiores a 10 salários-mínimos; os médicos em tal faixa salarial representavam apenas $21 \%$. O quadro altera-se nas faixas salariais mais altas (acima de 10 salários-mínimos) onde profissionais masculinos somam $78 \%$ enquanto os femininos $33 \%$ apenas.

É de fundamental importância frisar que, neste mesmo período, $50 \%$ das mulheres médicas tinham jornadas detrabalho acima de 50 horas, enquanto os médicos somavam apenas $30 \%$. O que faz cair por terra a hipótese de que jornadas de trabalho mais longas propiciam maiores rendimentos mensais. As razões de tal situação, certamente, não serão explicadas a partir desses dados*.

A situação da categoria dos enfermeiros é a seguinte: $100 \%$ dos enfermeiros ganhavam, em 1980, até 10 salários mínimos. As enfermeiras somavam $93 \%$ (já que na faixa salarial de 10 a 20 salários mínimos $7 \%$ delas se encontravam em tal faixa).

Apesar da categoria dos enfermeiros ser majoritariamente constituída por mulheres, verificou-se, em 1980, que $15 \%$ dessas percebiam salários inferiores a 3 salários mínimos; enquanto nesta situação, não há nenhum caso masculino. Interessante ressaltar que, mesmo numa categoria predominantemente feminina, a parcela de profissionais que encontram-se com salários baixíssimos é constituída exclusivamente de mulheres.

Já com o pessoal de enfermagem, a situação salarial tende a igualar-se, tanto para os homens como para as mulheres. Em 1980, $77 \%$ dos homens percebiam salários de até 3sm, enquanto as mulheres nesta situação somavam $85 \%$.

\section{CONSIDERAÇÕES FINAIS}

A forma de trabalho feminina em saúde, constitui hoje maioria absoluta no conjunto da FTS. Contudo, sabe-se que ela não se encontra em melhores condições de trabalho que a minoria masculina existente no setor.

Nas categorias aqui analisadas, verificou-se, por exemplo, que as mulheres assalariam-se mais que os homens, optam por jornadas de trabalho menores (em alguns casos) e têm salários bem inferiores ao dos homens.

No caso das médicas, estas acabam por especializar-se em áreas como: pediatria, ginecologia, psiquiatria, clínica médica, por exemplo. Estas especialidades dentro da medicina são, de um modo geral, consideradas de menor complexidade, menor prestrgio e conseqüentemente, com retorno financeiro mais baixo.

Cadernos de Saúde Pública, R.J., 2 (4) : 449-465, out/dez, 1986 
Essa recente inserção da mulher no mercado médico contribui sem dúvida para o processo de rejuvenescimento e feminilização desta categoria.

Por outro lado, a categoria de enfermagem de nível superior teve um sensível incremento da participação masculina. Considerada uma categoria essencialmente feminina de origem religiosa, esta, esteve sempre em desvantagem salarial se comparada a categoria médica. Seu baixo prestígio é, em parte, atribuído ao seu caráter assistencial voltado ao atendimento em hospitais filantrópicos, de caridade e religiosos, com funçöes essencialmente auxiliares ao trabalho médico*.

Constituindo, fundamentalmente, de mão-de-obra feminina, o pessoal de enfermagem (tanto os técnicos e auxiliares de enfermagem, quanto os atendentes) encontra-se em condições desfavoráveis no mercado (seus salários não ultrapassam a 3 salários mínimos). Registra-se nesta modalidade profissional a elevação do nivel de escolaridade, ou seja, há um crescente aumento da FT de nível médio (técnicos e auxiliares de enfermagem). Porém, seu baixo prestígio advém também da execução de tarefas rotinizadas, mais simplificadas, no auxílio as tarefas do enfermeiro. Este contingente de trabalhadores representa na estrutura hospitalar o que denomina-se de pessoal de linha. Aquele que executa tarefa da vida cotidiana do hospital.

E são exatamente nessas duas modalidades de profissionais (enfermeiros e pessoal de enfermagem) onde as funçōes dentro da estrutura hospitalar lhes atribuem menos prestígio e baixa remuneração, é que se tem a maior concentração de mulheres.

Apesar da crescente participação das mulheres no mercado de trabalho sabe-se que seu trabalho continua ainda a ser encarado pela sociedade como complementação da "renda familiar". Reforça-se este ponto de vista, quando observa-se que cerca de $70 \%$ da mão-de-obra feminina no setor saúde recebiam, em 1980, salários inferiores a 3 salários mínimos. Situação muito peculiar ocorre com os profissionais universitários. Em geral, as mulheres tendem a estacionar em níveis salariais mais baixos (atê 10 salários mínimos).

A questão da dupla jornada de trabalho, também deve ser mais bem avaliada, na medida em que isto em muito, contribui para a posição em desvantagem em que encontram-se as mulheres no mercado.

O problema da inserção da mulher no mundo do trabalho e da política, como já dito em trabalhos anteriores, $\varepsilon$ crucial para sua emancipação.

Para BUly ${ }^{1}$ é preciso encarar o trabalho feminino numa perspectiva estrutural para se definirem a quantidade e qualificação das mulheres a serem absorvidas pelo mercado de trabalho. E mais, a mulher acaba por preparar-se para tarefas consideradas femininas, aceita cargos considerados femininos e procura funções tidas como femininas; pedriatra, enfermei$\mathrm{ra}$, professora, faxineira, atendente, etc.

Cadernor de Saúde Pública, R.J., 2 (4) : 449-465, out/dez, 1986
* Para maiores crlticas ver: BARROS, UA. \& SILVA, M.R.F. A enfermagem e a mulherRecife, Centro de Estudos da Mulher, 1985. 
Por fim, é preciso que se empenhe na realização de trabalho de pesquisa de cunho mais qualitativo em instituições típicas da área de saúde, tais como: INAMPS, INPS, Hospitais públicos e privados, para precisar com maior clareza a situação real da expressiva FT feminina. Aí então será possível detectar níveis de discriminação e desvantagens de condições de trabalho em que se encontram as mulheres desse setor tão

importante da economia brasileira.

\section{BIBLIOGRAFIA CONSULTADA}

1. BLAY, E. A. O trabalho feminino. São Paulo, Centro de Estudos Rurais e Urbanos, 1973.

2. COSTA, M.A. Reproduçăo e mortalidade em cidade de porte médio (1960-1970). In: ENCONTRO NACIONAL DE ESTUDOS POPULACIONAIS, 2‥ São Paulo, 1980. Anais S ão Paulo, 1980.

3. MACHADO, M. H. A força de trabalho feminina em saúde no Brasil, 1970/1980. Belo Horizonte, 1986.

4. NOGUEIRA, R. P. Dinâmica do mercado de trabalho em saúde no Brasil, 1970/1983. Brasnlia, OPS, 1986.

5. NOGUEIRA, R.P. \& MEDICI, A.C. Caracteristicas demograficas da força de trabalho em saúde no Brasil, 1970 e 1980. Rio de Janeiro, 1983. mimeo. 